\title{
Does routine exercise induce stress and in consequence serum amyloid A (SAA) response in purebred Arabian racehorses?
}

\author{
Sylwester Kowalik and Ewa Tomaszewska \\ Department of Animal Physiology, Faculty of Veterinary Medicine, University of Life Sciences in Lublin, Poland
}

\begin{abstract}
Summary: This study aimed to evaluate whether a stress response to exercise increases the serum amyloid-A (SAA) production in purebred Arabian race horses. Eleven healthy Arabian horses aged 3 to 5 years were examined. All the horses were subjected to the same effort test consisting in $1200 \mathrm{~m}$ gallop at a speed typical for the daily training programme. Four venous blood samples were collected, namely: at rest (TO), immediately after the end of the exercise (T1), after 30 minutes of rest (T2), and after 24 hours from the first sampling (T3). Serum levels of ACTH, cortisol, and SAA were estimated by the ELISA method. To evaluate a relative intensity of exercise, the plasma lactate (LA) concentration and serum lactic dehydrogenase (LDH) activity were determined. The results obtained revealed that the type of exercise used caused a statistically significant short-term increase in the LA and LDH values. Similarly, the exercise induced a HPA axis response by a significant increase in ACTH and cortisol at T1 (Ps0.01). Then, the LA and hormones concentrations decreased significantly over one of the post-training sampling periods (T2) ( $P \leq 0.05$ and $P \leq 0.01$, respectively). No statistically significant differences were observed in the SAA serum level during the respective steps of the experiment (TO-T3). In conclusion, the exercise-induced stress clearly affected the HPA axis response in horses by transient raising the ACTH and cortisol concentration. In contrast, the routine training session did not promote significant changes in the SAA level in studied horses.
\end{abstract}

Keywords: ACTH, cortisol, horse, HPA axis, serum amyloid-A, stress

Citation: Kowalik S., Tomaszewska E. (2018) Does routine exercise induce stress, and in consequence serum amyloid A (SAA) response in purebred Arabian racehorses? Pferdeheilkunde 34, 121-125; DOI 10.21836/PEM20180203

Correspondence: Dr. Sylwester Kowalik PhD, Department of Animal Physiology, Faculty of Veterinary Medicine, University of Life Sciences in Lublin, ul. Akadedmicka 12, 20-033 Lublin, Poland; sylwester.kowalik@up.lublin.pl

\section{Introduction}

All mammals, including horses, respond to exercise by activating a wide array of behavioral and physiological mechanisms that are collectively referred as the stress response (Smith and Vale 2006, Cayado et al. 2006). The typical response consists of activation of the sympathetic nervous system, parasympathetic withdrawal, and increased activity of the hypothalamic-pituitary-adrenocortical (HPA) axis. Moreover, strenuous exercise causes the release of pro-inflammatory agents such as cytokines, prostaglandins, and certain proteins synthesized in the so-called acute phase response (APR) into the bloodstream (Cywińska 2012). The APR is thought to be a part of the early defense or innate immune system, which is triggered towards tissue injury, infection, inflammation, surgical trauma, or stress (Murata et al. 2004, Petersen et al. 2004, Eckersall and Bell 2010, Satué et al. 2013, Balikci et al. 2013). This non-specific, complex, and highly orchestrated inflammatory response is designed to reestablish homeostasis, promoting healing and repair processes (Crisman et al. 2008, Cray et al. 2009). A number of recent studies have reported that a reaction analogous to the APR in inflammatory conditions has been found after different kinds of exercise in humans, dogs, and horses (Cywińska et al. 2010, Wakshlag et al. 2010, Cywińska et al. 2012).

The response pattern of the APR is species-specific, especially for synthesis of acute phase proteins (APP), a class of proteins, whose plasma concentrations increase or decrease in animals subjected to external or internal challenges (Jacob- sen and Andersen 2007). In the horse, several proteins, including serum amyloid-A (SAA), fibrinogen, and haptoglobin, have acute phase properties (Cray et al. 2009, Casella et al. 2012). Among those, only SAA in equine practice is considered as the major APP, mainly because its level increases rapidly, even up to 1000 times during the APR, while the others will increase modestly up to 10 times of resting values (Jacobsen and Andersen 2007, Belgrave et al. 2013, Satué et al. 2013). A causal relationship between stress and APR has been confirmed, although the mechanism of APP response due to exercise-induced stress is yet to be clarified (Cray et al. 2009, Valle et al. 2015). The mechanism for stimulation of hepatic production of APP has been extensively studied; however, recent papers have revealed several discrepancies in the course of changes in SAA in horses that have undergone strenuous exercise. Kristensen et al. (2014) did not observe any changes in the plasma SAA level of Standardbred trotters after short-term acute exercise. Similarly, Cywińska et al. (2013) also did not observe significant differences in the plasma level of SAA in experienced purebred Arabian horses. In contrast, a recent study by Valle et al. (2015) has demonstrated a significant increase in the plasma level of SAA in Warmblood horses trained with a typical conditioning program. Similar changes in SAA release has been confirmed in exercised Thoroughbred horses (Turło et al. 2016). These findings, however, were not compared with the HPA axis activity in the studied horses. Moreover, according to research conducted by Kędzierski and Bergero (2006), it is known that Thoroughbred horses tolerate short-term, intensive exercise better than purebred Arabian horses. 
Therefore, the major assumption underlying this research was to clarify whether an exercise stressor can affect horse's metabolic processes by activation of both HPA axis and APR response. Thus, the aim of the present study was to evaluate if the exercise, used routinely in the race training of purebred Arabian horses, is a sufficiently strong stressor to elicit changes in the SAA level, the most useful marker of acute phase response in horses.

\section{Materials and Methods}

Horses

Eleven healthy purebred Arabian horses $(6$ stallions and 5 mares), aged 3 to 5 years, were used in the study. The horses were all stabled at the same training stable (Słuzewiec Racetrack, Warsaw, Poland), and each horse was kept in an individual box under the same environmental conditions. Prior to the study, the horses were routinely trained five days a week by the same trainer.

\section{Exercise test}

The study was performed in August, under clear weather conditions with a temperature of $18-20^{\circ} \mathrm{C}$, on a flat and dry sand racetrack. On the day of the test, the training session was performed in the morning, from 07:00 to 09:00 am. Initially, all horses had a 10-minute warm-up trot and canter with a rider. Then, they galloped on a $1200 \mathrm{~m}$ sand track at an average speed of $6.0 \mathrm{~m} / \mathrm{s}$, and at the finish, $800 \mathrm{~m}$ at a speed amounting to $12.8 \mathrm{~m} / \mathrm{s}$. Finally, they returned trotting to the stables (5 min). After the exercise, the horses were unsaddled and cooled down for 30 minutes on a mechanical horse walker. The training sessions were monitored using a portable GPS tracking system (Forerunner 305, Garmin, USA). During the study, the horses did not show clinical signs of any illness nor the mares showed external symptoms of estrus.

Four blood samples were taken at the following four phases: at rest, 30 minutes prior to the exercise (TO), immediately after the end of exercise (T1), 30 minutes after the end of the effort (T2), and after 24 hours from the first sampling (T3), before the next training session. The blood samples were collected from each horse by jugular venipuncture into two tubes: one containing EDTA, and other for serum separation. Plasma LA concentrations were determined within 15 minutes after the sampling. The remaining blood was centrifuged at $1000 \times G$ for 10 minutes and the serum supernatant was collected and frozen at $-20^{\circ} \mathrm{C}$ until further use.

\section{Laboratory analyses}

Serum ACTH and cortisol concentrations were determined using multi-species ELISA kits (DRG International Inc., Mountainside, NJ, USA). The SAA levels were measured using an ELISA test (Tridelta Development LTD, Ireland). All samples were analyzed in duplicate. The values of hormones and SAA were expressed as $\mathrm{ng} / \mathrm{ml}$. The level of blood LA was estimated by the spectrophotometric method using a Dr Lange enzymatic cuvette test (Warsaw, Poland) and expressed as mmol/l.
Serum LDH activity was measured using a Cormay diagnostic kit (Warsaw, Poland) and expressed as $U / l$.

\section{Statistics}

The results are presented as means \pm standard error of the mean (SEM). Statistical analyses were performed using the GraphPad Prism software package (Graph Pad Software, USA). Comparisons between the analyzed groups were made by the Student-Newman-Keuls test (ANOVA). The coefficient correlation was also determined to compare the exerciseinduced changes in the values of studied parameters. The statistical significance was accepted at the level of $P \leq 0.05$.

\section{Results}

The results of the measurement of studied hormones are presented in Fig. 1. Both the ACTH and cortisol concentrations increased significantly after the exercise (T1). The mean values of the serum ACTH level determined at T2 significantly decreased $(P \leq 0.05)$ but did not reach the level at the TO time. Similarly, the mean serum cortisol concentration significantly decreased $(P \leq 0.01)$ after 30 min of rest, but did not reach the values at $\mathrm{T} 0$. At $\mathrm{T} 3$, the mean concentrations of ACTH and cortisol returned to the initial levels. A significant correlation coefficient was found between the concentration of cortisol and $\mathrm{ACTH}$ in serum samples obtained at $\mathrm{T} 1$ $(r=0.64)$.

The mean values of blood LA and LDH obtained in the consecutive steps of the experiment are presented in Tab. 1. The statistical analysis revealed a significant increase in the blood LA concentration immediately after the exercise (T1) $(P \leq 0.05)$. Similarly, there was a significant increase of $L D H$ activity at $\mathrm{T} 1$ and $\mathrm{T} 2(\mathrm{P} \leq 0.01)$ in relation to T0 and T3.

Changes in the serum concentration of the SAA during the test are presented in Fig. 2. In general, the course of the changes in the SAA concentration was similar to that for ACTH and cortisol (Fig. 1). However, detailed analysis revea-

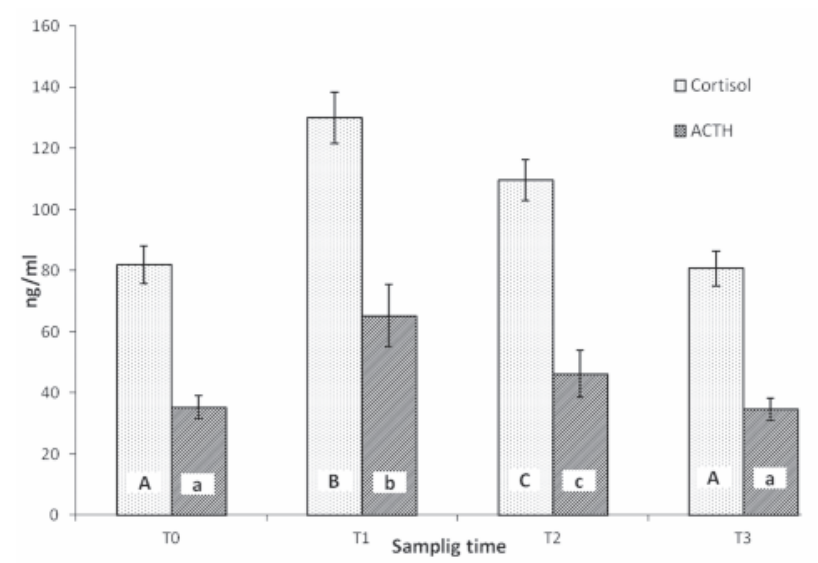

Fig. 1 Serum cortisol and ACTH concentrations during training session in Purebred Arabian racehorses (means \pm SE). TO - at rest, $\mathrm{T} 1$ - immediately after the end of exercise, T2 - 30 minutes after the end of the effort, T3 - 24 hours after the first sampling. A, B, C means marked with different superscripts differ at $p \leq 0.01 ; a, b, c-$ means marked with different superscripts differ at $p \leq 0.05$ 


\begin{tabular}{lcccc}
\hline Table 1 & Blood LA concentration and serum LDH activity in Purebred Arabian horses during training session (means \pm SE) & T2 & \\
\hline & T0 & T1 & $1.23 \pm 0.31^{a}$ & $0.94 \pm 0.13^{a}$ \\
\hline LA (mmol/l) & $0.92 \pm 0.15^{a}$ & $4.64 \pm 3.14^{b}$ & $417 \pm 28.20^{b}$ & $381 \pm 25.40^{a}$ \\
LDH (IU/l) & $379 \pm 26.60^{a}$ & $429 \pm 25.70^{b}$ &
\end{tabular}

T0 - at rest, T1 - immediately after the end of exercise, T2 - 30 minutes after the end of the effort, T3 - 24 hours after the first sampling $a, b$ - means marked with different superscripts differ at $p \leq 0.05$

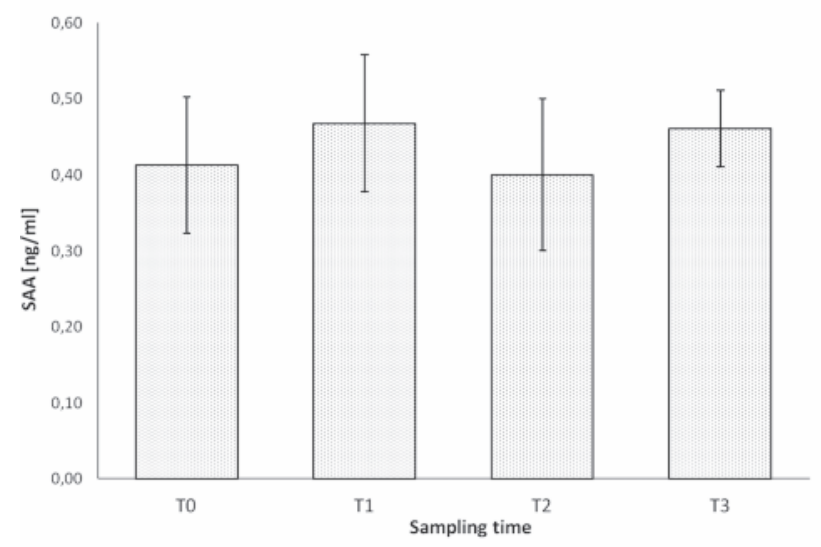

Fig. 2 Serum amyloid A concentration during training session in Purebred Arabian racehorses (means \pm SE). TO - at rest, T1 - immediately after the end of exercise, T2 - 30 minutes after the end of the effort, T3 - 24 hours after the first sampling, no statistical differences were found

led that there was no statistically significant differences between the levels of SAA during the respective steps of the experiment (TO-T3).

\section{Discussion}

The routine exercise induced transient increases in ACTH and cortisol concentrations in studied horses. Previous studies have shown that the intensity of exercise is the main factor that modulates the HPA axis response to effort (Cayado et al. 2006, Ferlazzo et al. 2012, von Lewiński et al. 2013). Therefore, to ensure an adequate exercise intensity of exercise performed by the studied horses, the plasma LA was determined. It is universally accepted that the rate of changes in circulating LA concentrations reflects the degree to which anaerobic metabolism contributes to energy production during exercise, thus the LA level in circulating blood is the reliable indicator of fatigue (Desmecht et al. 1996, Podolak et al. 2006, Kedzierski et al. 2014). As a first result, our analysis indicated that the horses developed a typical biochemical response to the exercise by increasing the LA concentration over the range of an anaerobic threshold $(4.0 \mathrm{mmol} / \mathrm{l})$. Therefore, it can be assumed that the physical effort applied during the training session was a sufficiently intense stressor to induce a local and/or systemic response. According to this, the exercise-induced stress elicited a systemic response and altered production of adrenal hormones.

Measurement of changes in the concentrations of ACTH and cortisol after a short-term high-intensity effort was shown to be effective for evaluating acute stress in racehorses (Fazio et al. 2008, Kędzierski et al. 2014). Also, the study by Ferlazzo et al. (2012) clearly has confirmed that participation in jumping competitions increased the circulatory levels of these hormones in horses. A simple but interesting experiment performed by Mircean and colleagues (2007) compared the influence of 4 different types of horse training (2 of moderate intensity and 2 of high intensity) on the LA and cortisol concentrations. Although the authors revealed a highly significant post-exercise increase in cortisol in all groups of horses, the most interesting finding was that the increased level of LA triggered HPA and cortisol secretion. This conclusion seems to be consistent with the results of our study. Moreover, our results indicated that the differences in the ACTH concentrations were correlated with the differences in cortisol secretion during the respective steps of the experiment. This is in line with the study by Ferlazzo et al. (2012), who have found that the peak of plasma ACTH in jumping horses occurs immediately after the end of the exercise and is associated with the cortisol plasma concentration.

In the present study, the positive significant correlation between the cortisol and ACTH serum concentration was noted only once, at the end of the exercise. This result allows us to postulate that the short-term medium-intensity effort applied in the study stimulates ACTH secretion; however, the cortisol response was not prolonged and did not increase after the end of the exercise. Consequently, the routine training session did not promote significant disturbances in the HPA axis in the trained purebred Arabian horses. In the light of this finding, it can be stated that the studied horses were well trained and/or the effort used in the routine training session was not a sufficient stressor to cause hormonal imbalance.

Despite the stated increase in ACTH and cortisol concentrations, SAA did not change significantly in the studied horses. The latest study by Turto et al. (2016) has revealed that a significant increase in plasma SAA in Thoroughbred racehorses occurs within 24 hours after the end of the race. However, as mentioned above, Thoroughbred racehorses and purebred Arabian horses differ in terms of their exercise capacity, and therefore the results obtained are not fully comparable between these breeds (Kędzierski and Bergero 2006). In general, in general, the intensity of effort during the race is much higher than that used in daily training sessions (Desmecht et al. 1996). Moreover, an APR develops in horses within several hours after exposure to acute or continuous stressors and reach peak values only after 36-48 hours (Casella et al. 2012, Satué et al. 2013). However, we did not observe statistically significant changes in the SAA level during all the respective steps of the experiment. This may be attributed to the fact that APR required a longer and/or stronger effort to increase the SAA in blood serum. This statement is consistent with the results of several studies which examined the effect of high-intensity exercise on the plasma SAA concentration in horses undergoing different types of training. Larsson et al. (2013) have reported that the SAA concentration increase significantly by more than $1800 \%$, in horses participating in a $120 \mathrm{~km}$ endurance ride, in comparison to the resting values. 
Similar studies have been conducted by Cywińska and colleagues $(2010,2013)$. The authors have revealed that the plasma SAA concentration in Arabian horses increase significantly after the endurance rides at the distances of 80, 120, and $160 \mathrm{~km}$. Moreover, they have found a correlation between the pre-race SAA level and the physical status of the horses, i.e. if the basic values of SAA were high, the horses showed worst physical function and often did not end of the race.

In our study, the concentration of SAA measured in accordance with experimental assumptions showed no significant changes. The lack of changes observed immediately after the exercise could be initially explained by the selected sampling time, i.e. approximately 5 minutes after the end of exercise, which was most likely too early to observe an increase in SAA concentration. However, the SAA level in the consecutive blood serum samples, including the T3 time, did not increase either. According to this result it seems that the routine training session did not promote the SAA systemic response. One of the most likely cause of these results is that the Arabian horses subjected to the experiment were well trained and, therefore, the daily routine training did not affect the APR reaction. Finally, it should be underlined that all the experimental horses were healthy and clinically normal, and completed the training without any injures. This statement seems to be essentially methodological in estimation of plasma SAA of exercised horses. Especially, an intensive acute exercise has detrimental effects on defense mechanisms, which finally leads to tissue lesions (Kinnunen et al. 2005, Capelli et al. 2008). The resulting lesions, in turn, can cause, as an overlapping factor, additional secretion of SAA into bloodstream (Turlo et al. 2015). It has been reported previously that $\mathrm{LDH}$ correlates well with injuries that occur in horses during a wide variety of exercise activities (Chiaradia et al. 1998, Muñoz et al. 2002, TeixeiraNeto et al. 2008). Our findings on the LDH concentration during the training session showed no significant differences between the values obtained at rest and $24 \mathrm{~h}$ after the exercise. This means that there were no serious tissue fatigue injuries in the horses, despite the intensity of the exercise performed.

In conclusion, our study indicated that although routine race training sessions of purebred Arabian horses activate the HPA axis response, they did not promote significant changes in the SAA level.

\section{Animals welfare statement}

All procedures were approved by the Local Ethical Review Committee for Animal Experiments in Lublin (45/2017) and conducted according to the European Community regulations concerning the protection of experimental animals.

\section{References}

Balikci E., Yildiz A., Gurdogan F. (2013) Selected acute phase proteins, oxidative stress biomarkers, and antioxidants in aborting and non-aborting goats infected with border disease virus. Bull. Vet. Inst. Pulawy 57, 371-374; DOI 10.2478/bvip-2013-0064

Belgrave R. L., Dickey M. M., Arheart K. L., Cray C. (2013) Assessment of serum amyloid $A$ testing of horses and its clinical application in a specialized equine practice. J. Am. Vet. Med. Assoc. 243, 113-119; DOI 10.2460/javma.243.1.113
Cappelli K., Felicetti M., Capomaccio S., Spinsanti G., Silvestrelli M., Supplizi A. V. (2008) Exercise induced stress in horses: Selection of the most stable reference genes for quantitative RT-PCR normalization. BMC Mol. Biol. 9, 49-56; DOI 10.1186/14712199-9-49

Casella S., Fazio F., Giannetto C., Giudice E., Piccione G. (2012) Influence of transportation on serum concentrations of acute phase proteins in horse. Res. Vet. Sci. 93, 914-917; DOI 10.1016/i.rvsc.2012.01.004

Cayado P., Muñoz-Escassi B., Dominguez C., Manley W., Olabarri B., Sánchez de la Muela M., Castejon F., Marañon G., Vara E. (2006) Hormone response to training and competition in athletic horses. Equine Vet. J. Suppl. 36, 274-278; DOI 10.1111/i.20423306.2006.tb05552.x

Chiaradia E., Avellini L., Rueca F., Spaterna A., Porciello F., Antonioni M. T., Gaiti A. (1998) Physical exercise, oxidative stress and muscle damage in racehorses. Comp. Biochem. Physiol. B 119 , 833-836

Cray C., Zaias J., Altman N. H. (2009) Acute phase response in animals: a review. Comp. Med. 59, 517-526

Crisman M. V., Scarratt W. K., Zimmerman K. L. (2008) Blood proteins and inflammation in the horse. Vet. Clin. North Am. Equine Pract. 24, 285-297; DOI 10.1016/i.cveq.2008.03.004

Cywińska A., Witkowski L., Szarska E., Schollenberger A., Winnicka A. (2013) Serum amyloid A (SAA) concentration after training session in Arabian race and endurance horses. BMC Vet. Res. 9, $91-$ 97; DOI 10.1186/1746-6148-9-91

Cywińska A., Gorecka R., Szarska E., Witkowski L., Dziekan P., Schollenberger A. (2010) Serum amyloid A level as a potential indicator of the status of endurance horses. Equine Vet. J. Suppl. 38, 23-27; DOI 10.1111/i.2042-3306.2010.00280.x

Cywińska A., Szarska E., Gorecka R., Witkowski L., Hecold M., Bereznowski A., Schollenberger A., Winnicka A. (2012) Acute phase protein concentrations after limited distance and long distance endurance ride in horses. Res. Vet. Sci. 93, 1402-1406; DOI 10.1016/i.rvsc.2012.02.008

Desmecht D., Linden A., Amory H., Art T., Lekeux P. (1996) Relationship of plasma lactate production to cortisol release following completion of different types of sporting events in horses. Vet. Res. Commun. 20, 371-379

Eckersall P. D., Bell R. (2010) Acute phase proteins: Biomarkers of infection and inflammation in veterinary medicine. Vet. J. 185, 2327; DOI 10.1016/j.tvjl.2010.04.009

Fazio E., Medica P., Cravana C., Ferlazzo A. (2008) Effects of competition experience and transportation on the adrenocortical and thyroid responses of horses. Vet. Rec. 163, 713-716

Ferlazzo A., Medica P., Cravana C., Fazio E. (2012) Circulating endorphin, adenocorticotropin, and cortisol concentrations of horses before and after competitive show jumping with different fence heights. J. Equine Vet. Sci. 32, 740-746; DOI 10.1016/i.jevs. 2012.02.021

Jacobsen S., Andersen P. H. (2007) The acute phase protein serum amyloid $A(S A A)$ as a marker of inflammation in horses. Equine Vet. Educ. 19, 38-46; DOI:10.1111/i.2042-3292.2007.tb00 550.x

Kędzierski W., Bergero D. (2006) Comparison of plasma biochemical parameters in Thoroughbred and Purebred Arabian horses during the same-intensity exercise. Polish J. Vet. Sci. 9, 233-238

Kędzierski W., Cywińska A., Strzelec K., Kowalik S. (2014) Changes in salivary and plasma cortisol level in Purebred Arabian horses during race training session. Animal Sci. J. 85, 313-317; DOI 10.1111 /asj. 12146

Kinnunen S., Hyyppä S., Lappalainen J., Oksala N., Verojärvi M., Nakao C., Hänninen O., Sen C.K., Atalay, M. (2005) Exerciseinduced oxidative stress and muscle stress protein responses in trotters. Eur. J. Appl. Physiol. 93, 496-501; DOI 10.1007/s00 421-004-1162-x

Kristensen L., Buhl R., Nostell K., Bak L., Petersen E., Lindholm M., Jacobsen S. (2014) Acute exercise does not induce an acute phase response (APR) in Standardbred trotters. Canadian J. Vet. Res. 78, 97-102 
Larsson J., Pilborg P. H., Johansen M., Christophersen M. T., Holte A., Roepstorff L., Olsen L. H., Harrison A. P. (2013) Physiological parameters of endurance horses pre-compared to post-race, correlated with performance: a two race study from Scandinavia. ISRN Vet. Sci. 18, 1-12; DOI 10.1155/2013/684353

Mircean M., Giurgiu G., Mircean V., Zinveliu E. (2007) Serum cortisol variation of sport horses in relation with the level of training and effort intensity. Bull. Univ. Agric. Sci. Vet. Med. Cluj-Napoca 64, 488-492

Muñoz A., Riber C., Santisteban R., Lucas R. G., Castejón F. M. (2002) Effect of training duration end exercise on blood-borne substrates, plasma lactate and enzyme concentrations in Andalusian, Anglo-Arabian and Arabian breeds. Equine Vet. J. Suppl. 34, 245-251; DOI 10.1111/j.2042-3306.2002.tb05427.x

Murata H., Shimada N., Yoshioka M. (2004) Current research on acute phase proteins in veterinary diagnosis: an overview. Vet. J. 168, 28-40; DOI 10.1016/S1090-0233(03)00119-9

Petersen H. H., Nielsen J. P., Heegaard P. M. H. (2004) Application of acute phase protein measurements in veterinary clinical chemistry. Vet. Res. 35, 163-187; DOI 10.1051/vetres:2004002

Podolak M., Kédzierski W., Bergero D. (2006) Comparison of the blood plasma catecholamines level in Thoroughbred and Arabian horses during the same-intensity exercise. Polish J. Vet. Sci. 9, 71 73

Satué K., Calvo A., Gardón J. C. (2013) Factors influencing serum amyloid type A (SAA) concentrations in horses. Open J. Vet. Med. 3, 58-66; DOI 10.4236/ojvm.2013.31010

Smith S. M., Vale W. W. (2006) The role of the hypothalamic-pituitary-adrenal axis in neuroendocrine responses to stress. Dialogues Clin. Neurosci. 8, 383-394
Teixeira-Neto A. R., Ferraz G. C., Moscardini A. R. C., Balsamão G. M., Souza J. C. F., Queiroz-Neto A. (2008) Alterations in muscular enzymes of horses completing long-distance endurance rides under tropical climate. Arq. Bras. Med. Vet. Zootec. 60, 543-549; DOI 10.1590/S0102-09352008000300004

Turło A., Cywińska A., Czopowicz M., Witkowski L., Jaśkiewicz A., Winnicka A. (2016) Racing induces changes in the blood concentration of serum amyloid $A$ in Thoroughbred racehorses. J. Equine Vet. Sci. 36, 15-18; DOI 10.1016/i.jevs.2015.09.008

Turło A., Cywińska A., Czopowicz M., Witkowski L., Szarska E., Winnicka A. (2015) Post-exercise dynamics of serum amyloid A blood concentration in thoroughbred horses classified as injured and non-injured after the race. Res. Vet. Sci. 100, 223-225; DOI 10.1016/j.rvsc.2015.04.008

Valle E., Zanatta R., Odetti P., Traverso N., Furfado A., Bergero D., Badino P., Girardi C., Miniscalco B., Bergagna S., Tarantola M., Intorre L., Odore R. (2015) Effects of competition on acute phase proteins and lymphocyte subpopulations - oxidative stress markers in eventing horses. J. Anim. Physiol. Anim. Nutr. 99, 856-863; DOI 10.1111/ipn. 12289

von Lewinski M., Biau S., Erber R. Ille N., Aurich J., Faure J-M., Möstl E., Aurich C. (2013) Cortisol release, heart rate and heart rate variability in the horse and its rider: Different responses to training and performance. Vet. J. 197, 229-232; DOI 10.1016/i.tvil. 2012.12.025

Wakshlag J. J., Stokol T., Geske S. M., Greger C. E., Angle C. T., Gillette R. L. (2010) Evaluation of exercise - induced changes in concentration of $\mathrm{C}$-reactive protein and serum biochemical values in sled dogs competing a long-distance endurance race. Am. J. Vet. Res. 71, 1207-1213; DOI 10.2460/ajvr.71.10.1207 